\title{
Trabajo Social y resiliencia frente a casos de embarazo en adolescentes de la Unidad Educativa Picoazá
}

\author{
Social work and resilience in the event of pregnancy in adolescents of the Picoaza \\ educational unit
}

Tania Liceth Zamora Zambrano' (iD (8), Eva Margarita Alcívar Medranda 2 (iD) (8)

\author{
Cómo citar \\ Zamora, T. y Alcívar, E. (2021). Trabajo social y resiliencia frente a casos de embarazo en adolescentes de la unidad educativa \\ Picoaza. Socialium, 5(2), 204-216. https://doi.org/10.26490/uncp.sl.2021.5.2.933
}

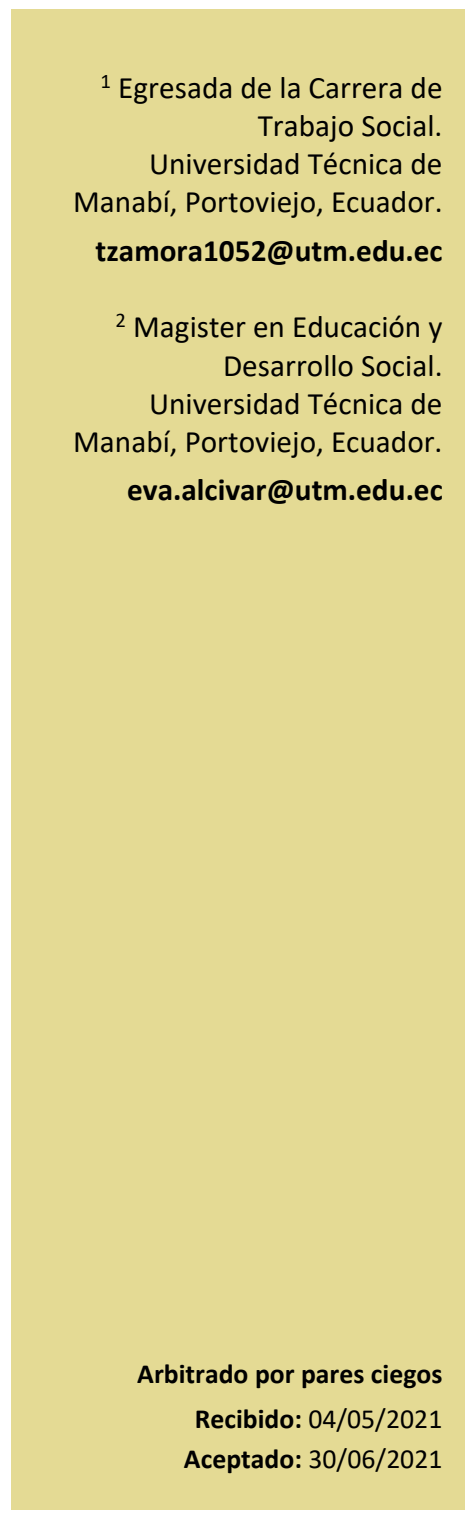

\section{RESUMEN}

El trabajo social, como disciplina que en sus prácticas afronta la problemática del embarazo adolescente en forma integral. El objetivo fue identificar el trabajo social y resiliencia frente casos de embarazo en adolescentes de la unidad educativa Picoaza, Ecuador. La investigación consideró el método científico, deductivo y analítico, nivel descriptivo con enfoque mixto, se utilizó como instrumento la guía de entrevista y la Escala de Resiliencia SV-RES de Eugenio Saavedra Guajardo y Marcos Villalta Paucar la cual se aplicó de manera online a la trabajadora social del Departamento de Consejería Estudiantil DECE y a estudiantes embarazadas. De acuerdo a los resultados, el $53 \%$ de estudiantes se considera con valores, lo que permite identificar un adecuado estado de aceptación, mientras que con el $27 \%$ un importante grupo se considera temeroso o débil, lo que puede dar pautas a posibles desajustes de comportamiento, con el $13 \%$ indican ser valiente y tener fortaleza y con el $7 \%$ se consideran optimista ante cualquier cambio en sus vidas. Por otro lado, las acciones que desarrolla la profesional en trabajo social de la unidad educativa, deben ser coordinadas con el equipo técnico profesional del departamento de bienestar estudiantil, quienes en conjunto desarrollen estrategias, que fomenten la autoestima.

Palabras clave: embarazo adolescente; trabajo social; resiliencia en estudiantes; intervención profesional.

\section{ABSTRACT}

Social Work is one of the disciplines whose practices address the problem of adolescent pregnancy in a comprehensive manner. The objective was to identify Social Work and resilience in cases of pregnancy in adolescents in the educational unit Picoaza, Ecuador. The research considered the scientific, deductive and analytical method, descriptive level with qualitative approach, the interview guide and the Resilience Scale SV-RES of Eugenio Saavedra Guajardo and Marcos Villalta Paucar were used as an instrument, which was applied online to the Social Worker of the Student Counseling Department DECE and pregnant students. According to the results, $53 \%$ of students consider themselves to have values, which allows identifying an adequate state of acceptance, while with $27 \%$ an important group considers themselves fearful or weak, which can give guidelines to possible behavioral imbalances, with $13 \%$ they indicate being brave and having strength and with $7 \%$ they consider themselves optimistic before any change in their lives. On the other hand, the actions developed by the social work professional of the educational unit should be coordinated with the professional technical team of the student welfare department, who together develop strategies to promote self-esteem.

Keywords: adolescent pregnancy; social work; student resilience; professional intervention. 


\section{Introducción}

El embarazo es un proceso natural y especial en la vida de cada mujer, el cual comprende múltiples cambios, tanto físicos y fisiológicos en el interior del útero materno, es por ello que la madre requiere comprensión y adaptación a los cambios fisiológicos y psicológicos, según la (Organización Mundial de la Salud, 2006, p. 13) "el embarazo es el período que transcurre desde la implementación del óvulo fecundado en el útero hasta el momento del parto, el cual contiene una duración de 40 semanas." Es importante considerar que es una etapa importante en la mujer, ya que en la especie humana la gestación suele ser única, por lo cual necesita cuidados, ya que el proceso ocurre paso a paso, el cual permita identificar de manera oportuna el desarrollo óptimo del embarazo.

Así mismo, como lo resaltan Balestena et al. (2015) "el embarazo y el parto son considerados como fisiológicos y totalmente naturales, además son recibidos como un regalo al sexo femenino, sin embargo, en determinadas circunstancias y condiciones requieren de cuidados específicos para evitar riesgos de mortalidad materna" (p. 3). Por este motivo se considera al embarazo como el periodo que marca la vida de una mujer, y sin bien es cierto que representa una experiencia vital y en cierta media una realización plena que trae consigo emociones, incógnitas y decisiones que tomar con responsabilidad con el fin de preservar en todo momento la salud maternal y fetal.

Por otro lado, Martínez et al. (2018), establecen que "durante el embarazo, se presentan una serie de alteraciones hormonales que llevan a cambios fisiológicos en diferentes sistemas y entre estos cambios pueden implicar riesgos que conllevan diferentes trastornos, entre ellos la anemia, obesidad y riesgos de morbilidad" (p. 2).

Esto constituye un desafío que se complica en la actualidad, ya que en este punto el embarazo muestra un factor de riesgo mucho mayor para la madre y para el niño es por ello, que se debe detectar enfermedades en estadios clínicos para evitar un alto índice de mortalidad.

Si bien, es cierto que el embarazo es un proceso natural del organismo femenino, por esta razón, la reproducción asistida ha dado pasos gigantes durante la última década, siendo considerado favorable para la mujer y el recién nacido, No obstante, se debe tomar en cuenta que cuando ocurre lo contrario, conceptualmente tenemos instalado un embarazo de alto riesgo.

No obstante, se debe destacar la importancia que tienen los Trabajadores Sociales en la intervención con adolescentes embarazadas, acción que día a día tiene relevancia y sobre la cual existen limitados conocimientos por la sociedad. Para esto se deben estimar que la adolescencia es un período de cambios biológicos, sociales y psicológicos constantes, en donde interactúan múltiples factores que influyen en la vida social del adolescente de diferentes maneras, considerando el medio en que se desarrolla de manera 
cotidiana la vida normal del adolescente, la coyuntura socioeconómica la cual se considera un elemento condicionante al observar las oportunidades, en donde son escasas.

En este orden de ideas, se puede indicar que el embarazo adolescente se puede considerar uno de los factores de mayor fragilidad en los sectores de escasos recursos. Pues al existir limitado conocimiento sobre el tema de la realidad nacional y local, se pone al adolescente en una situación compleja de verdadera desprotección.

Dadas las condiciones que anteceden en esta investigación es importante abordarla desde un enfoque que permita la comprensión desde un carácter social amplio, que impulse una contextualización teórica y al mismo tiempo metodológica, es así que el objetivo de esta investigación es identificar el trabajo social y Resiliencia frente casos de embarazo en adolescentes de la unidad educativa Picoaza.

\section{Embarazo en adolescentes}

Hoy en día, son más las mujeres que quedan embarazadas a partir de 14 a 16 años, son en su mayoría embarazos no planificados, es por ello, que los embarazos constituyen unos de las principales preocupaciones de la conducta sexual de los jóvenes, Urbina y Pacheco $(2006$, p. 119) manifiestan que "Hay más de mil millones de adolescentes, 85 \% en los países en desarrollo, más de la mitad ha tenido relaciones sexuales antes de los 16 años". En este sentido, es importante considerar que se debe tener en cuenta al adolescente e incentivar en ellos la responsabilidad que implica llegar a ese estado, para ello es impredecible la existencia de charlas que contribuyan a la comprensión de la realidad en la cual pueden llegar si no se toman medidas de prevención adecuadas.

Se hace necesario resaltar que el embarazo durante la adolescencia es un fenómeno que ha llamado la atención mundial desde el siglo pasado, para la Organización Mundial de la Salud (2006, p. 12), la adolescencia se concibe como "la etapa comprendida entre los 10 a los 19 años, esto presenta un problema de salud pública y un periodo crítico en el desarrollo de los comportamientos sexuales que pueden llevar a adquirir un embarazo no deseado".

Por lo tanto, se examina un incremento de la actividad sexual en adolescentes consecuencia de la desinformación y el uso inadecuado o no uso de los métodos anticonceptivos. Por su parte, León et al. (2008, p. 43) definen el embarazo adolescente como "la gestación en mujeres, cuyo rango de edad se considera adolescente, independiente de su edad ginecológica". Es importante considerar que en nuestra sociedad la edad considerada adolescente tiene relación con su fisiología para ello, se puede caer en equivocaciones por el simple hecho de observar los rasgos de una joven y con ello determinar que puede ya estar apta para esa condición, según informa Barón $(2013$, p. 68) “el embarazo en adolescentes es una situación conflictiva no solo para el individuo sino también para la familia y la sociedad que lo rodea porque implica factores socioculturales y económicos que comprometen el binomio madre e hijo". Es 
decir que, de acuerdo a lo expresado, el embarazo en adolescentes es consecuencia de una práctica sexual casi siempre irresponsable y ocurre en todas las franjas sociales, pero prevalece en aquellos sectores de menor nivel socioeconómico y en consecuencia en los países en desarrollo.

\section{Embarazo infantil}

Caballero y Méndez (2005, p. 62) mencionan que "el embarazo infantil es una problemática de nivel mundial y que aqueja a todas las sociedades y gobiernos; en los últimos 10 años se ha incrementado las cifras de nacimiento en niñas en edades de 10 años" desde esta perspectiva el embarazo o gestación infantil causa mucho desasosiego en la sociedad, en este orden de ideas, Gómez et al. (2010, p. 14) establecen que "la pobreza influye en la probabilidad que tienen las niñas de quedar embarazadas y si es así entran en un círculo vicioso, ya que la maternidad precoz suele comprometer sus resultados académicos y su potencial económico". Por ello, es importante destacar que la falta de recursos y de oportunidades educativas $y$, por ende, laborales son unos de los efectos negativos que conllevan un embarazo.

Según lo expresado, la mayor parte de estos embarazos no son planeados ni deseados y ocurre en todos los estratos sociales originado situaciones complicadas, para Posada (2004, p. 5) "en el ámbito biológico, la joven presenta alto riesgos durante el embarazo o parto ya que su cuerpo no se encuentra totalmente desarrollado para los futuros cambios físicos, por lo tanto, se le debe de brindar atención médica por el bienestar de su salud y el del niño".

Existen diversos factores inciden en esta problemática, como el desconocimiento del cuerpo humano y de la reproducción, por otra parte, puede implicar un mayor riesgo como deducción de una violación o en efecto de abuso sexual por parte de un familiar o de un conocido, frente a esta realidad surge la necesidad de implementar medidas para prevenir embarazos precoces, como la educación sexual y el apoyo de padres de familia los cuales cumplen un importante rol.

Atendiendo estas consideraciones, el lazo entre embarazo en la adolescencia y pobreza es una de las cuestiones centrales en torno al cual rueda el estudio de la maternidad en adolescentes, desde esta perspectiva Binstock y Náslund (2013, p. 26) señalan que "el embarazo en la adolescencia tiene una repercusión importante en el índice de deserción escolar, reincidencia en embarazos no deseados, el cual se considera un problema de salud pública de alto impacto negativo". Es así que comprender las repercusiones que tiene el embarazo a temprana edad, puede resultar un desafío para los jóvenes, puesto que esto implica anticipar las consecuencias que pueden suscitar con ello.

\section{Trabajador social}

El embarazo a temprana edad se lo puede comprender como tema de interés, pues genera preocupación, en especial para el profesional en Trabajo Social, puesto que es quien interviene desde las unidades 
educativas, para evitar la deserción escolar por esta causa: "el trabajo social forma parte del sistema educativo formal y juega un papel importante en la intervención preventiva y asistencial en temas como el absentismo y el fracaso educativo, la integración de inmigrantes y colectivos desfavorecidos, la detección de malos tratos" (Fernández et al., 2002, p. 67). Desde esta perspectiva, el trabajador Social debe tener valores y actitudes para enfrentar los problemas de un grupo e incluso de la sociedad misma que existen en los centros educativos del nivel medio por los embarazos en adolescentes.

Es en este punto donde surge el embarazo adolescente consecuencia de una práctica sexual casi siempre irresponsable. Por ello los padres deben brindar apoyo y tener la colaboración de un trabajador social para que socialicen a sus hijos respecto al tema de la sexualidad en general y el uso de métodos anticonceptivos, en referencia a lo mencionando anteriormente, los padres deben considerar en todo momento el futuro de los hijos para que estos puedan sentir el apoyo por parte de sus familiares, y la sociedad.

\section{Método}

Tipo de estudio. Para realizar la ejecución de esta investigación se utilizó el diseño no experimental, el cual se apoyó con el método analítico, descriptivo, cuantitativo y bibliográfico; los cuales permitieron contextualizar los aportes de la investigación para que los recursos obtenidos se empleen como material de apoyo y con ello sirvan de soporte bibliográfico. También se aplicó el método lógico científico: inductivo, deductivo, enfoque mixto, lo que permitió analizar la información obtenida y valorar aquella de utilidad priorizando el objetivo de este estudio

Población y muestra. En este estudio se consideró una población de estudiantes del tercero de bachillerato de la Unidad Educativa Picoaza, para efectos, se utilizó una muestra de 15 estudiantes en estado de embarazo

Procedimientos de la recolección de datos. Para recolección de datos se utilizó como instrumento la guía de entrevista y la Escala de resiliencia SV-RES de los autores Eugenio Saavedra Guajardo y Marcos Villalta Paucar la cual se aplicó de manera online a trabajadoras sociales del Departamento de Consejería Estudiantil DECE utilizando la plataforma WhatsApp, la cual permitió realizar diferentes cuestiones en relación con la problemática investigada, mientras que a estudiantes embarazadas de la unidad educativa Picoaza, se les aplicó un cuestionario de encuesta Semiestructurado con preguntas lo que concedió cumplir con el objetivo expuesto en este trabajo.

Procedimiento en la recolección de datos. Se empleó el procedimiento de esta investigación con la aplicación de la técnica de entrevista estructurada, la cual se direccionó en identificar acciones realizadas por la profesional en Trabajo Social desde el DECE (Departamento de Consejería estudiantil), por lo que 
se identificó que al existir limitaciones por la pandemia del COVID-19 la mejor alternativa consistía en realizarla utilizando los recursos tecnológicos existentes. No obstante, en la encuesta que se aplicó se elaboró mediante el recurso de Google Forms, lo que permitió enviarles un enlace por medio de correo electrónico directamente a las estudiantes en estado de embarazo para recopilar la información presentada en este estudio.

Aspectos éticos. Esta investigación consideró importante reservar los nombres de la población objeto de estudio como medida de protección la privacidad, no obstante, a las estudiantes embarazadas se les explicó que este trabajo era con fines académicos por cuanto se garantiza la no divulgación de datos sensibles o de carácter reservados.

Análisis de datos. El análisis se realizó producto de la información proporcionada en este estudio, por estudiantes embarazadas en la que se procesó utilizando el programa Microsoft Excel obteniendo los porcentajes de acuerdo a las cuestiones establecidas en la encuesta, comparando los factores de la escala de resiliencia asociados con los resultados, considerando los 12 ítems que establecen Marcos Villalta Paucar, mientras en la entrevista se evidenció las acciones que desde el Trabajo Social desarrollan cuando se presenta alguna problemática dentro de las instalaciones académicas.

\section{Resultado}

De acuerdo con la entrevista realizada a la profesional en Trabajo Social del Departamento de consejería estudiantil, existe preocupación por el incremento de estudiantes que se embarazan si haber concluido su formación profesional, lo cual conlleva a cambiar de planes en su vida, Ella mencionó:

Existe preocupación por las autoridades del plantel al evidenciar el aumento considerado de estudiantes en estado de embarazo, lo que se atribuye que puede incidir en su proceso formativo, puesto que si bien, no es un impedimento tener un hijo para continuar sus estudios, esto si puede afectar su estado emocional, por el proceso biológico que implica transformación en el cuerpo de las estudiantes.

Del mismo modo indicó que:

En investigaciones llevada a cargo por ese departamento se han constatado cambios en el comportamiento de estudiantes, que por coincidencia están en estado de gestación, lo que se atribuye a posibles problemas en el entorno familiar. Por último, menciona su preocupación al existir ausentismo por parte de este grupo de jóvenes, no obstante, acusa esto a la situación mundial a causa del COVID-19. Dichas acciones son en conjunto realizada por la psicóloga clínica que establece los procedimientos de intervención en estos casos. 
Para continuar con el procedimiento metodológico se aplicó una encuesta a estudiantes embarazadas considerando los factores de la escala de Resiliencia SV - RES la cual desarrolló Saavedra y Villalta (2007), fundamentada en el modelo de Grotberg (1995). Por ello tomando como referencia (ver tabla 1) se establecen ítems en esta investigación que van con relación a los factores resilientes.

\section{Tabla 1}

Niveles de estructuración de la conciencia

\begin{tabular}{lcccc}
\hline $\begin{array}{l}\text { Competencias } \\
\text { interacciónales de } \\
\text { Grotberg (1995) }\end{array}$ & Condición de base & Visión de sí mismo & Visión del problema & Respuesta resiliente \\
\hline Yo soy, yo estoy & F1: Identidad & F2: Autonomía & F3: Satisfacción & F4: Pragmatismo \\
Yo tengo & F5: Vínculos & F6: Redes & F7: Modelos & F8: Metas \\
Yo puedo & F9: Afectividad & F10: Autoeficacia & F11: Aprendizaje & F12: Generatividad
\end{tabular}

Nota. Elaborado de Villalta Paucar; Factores asociados al rendimiento académico en estudiantes de contextos de alta vulnerabilidad social (2010).

En la tabla anterior se identifican 12 factores de los cuales el Factor 1: se relaciona con Identidad. (Yo soycondiciones de base). Hace referencias a juicios de carácter general tomados de los aspectos culturales que precisan al sujeto de un modo determinado. El factor 2: hace referencia a la Autonomía. (Yo soy/yo estoy-visión de sí mismo). Describe los juicios sobre el lazo que el sujeto establece consigo mismo, mientras que el factor 3: indica la satisfacción individual. (Yo soy, yo estoy, visión del problema). Hacen referencia a un estado de satisfacción personal, efectos de logro y autovaloración. El factor 4: establece el pragmatismo. (Yo soy, yo estoy-respuesta resiliente). Puede indicar un sentido práctico como necesidad de interpretar varias acciones que realizan, a su vez el Factor 5: establece vínculos. (Yo tengo, condiciones de base). Hace referencia a las relaciones cercanas e intensas con vínculos en la infancia que especifican estructuras de apego las cuales se orientan sistemas de creencias o aspectos religiosos (Saavedra, 2003). Factor 6: se relaciona con redes. (Yo tengo, visión de sí mismo). Concierne lo afectivo que establece la persona con su entorno social cercano, por otro lado, el Factor 7: indica modelos. (Yo tengo, visión del problema). Aquí se especifican juicios que refieren a personas y situaciones que sirven de orientación al sujeto para hacer frente a sus problemas, mientras que el factor 8: determina las metas. (Yo tengorespuesta resiliente). Este tiene relevancia al sentido de la acción para determinar una situación problemática.

Sin embargo, el factor 9: Afectividad. (Yo puedo-condiciones de base). Se relaciona a la valoración positiva de la vida emocional y el factor 10: Autoeficacia. (Yo puedo visión de sí mismo). Establece las visiones de éxito lo que permite a la persona reconocer en sí mismo un potencial ante una situación problemática. Factor 11: se relaciona con el aprendizaje. (Yo puedo-visión del problema). Permite aprovechar la experiencia coexistida, para aprender de los errores y evaluar el propio actuar para corregir la acción 
negativa. Factor 12: establece la generatividad. (Yo puedo, respuesta resiliente). Determina la relevancia con la capacidad de pedir ayuda a los demás para así solucionar distintas situaciones problemáticas.

Tabla 2

Cómo se considera usted, dirigido a estudiantes embarazadas de la Parroquia Picoaza, Ecuador 2021

\begin{tabular}{lcc}
\hline Ítems & F & $\%$ \\
\hline Temeroso /débil & 4 & 27 \\
Valiente /fuerte & 2 & 13 \\
Con valores /principios & 8 & 53 \\
Optimista & 1 & 7 \\
Total & 15 & 100 \\
\hline
\end{tabular}

Nota. Elaboración propia en base a cuestionario aplicado a estudiantes embarazadas de Unidad Educativa Picoaza, Ecuador.

De acuerdo a estos resultados el $53 \%$ de estudiantes embarazadas indica que se consideran con valores, lo que permite identificar un adecuado estado de aceptación, mientras que con el $27 \%$ un importante grupo se considera temeroso o débil, lo que puede dar pautas a posibles desajustes de comportamiento, con el $13 \%$ indican ser valiente y tener fortaleza y con el $7 \%$ se consideran optimista ante cualquier cambio en sus vidas.

\section{Tabla 3}

En que ocupa la mayor cantidad de su tiempo, dirigido a estudiantes embarazadas de la Parroquia Picoaza, Ecuador 2021

\begin{tabular}{llc}
\hline Ítems & F & $\%$ \\
\hline En actividades académicas & 3 & 20 \\
Quehacer Domestico & 5 & 33 \\
Ocio & 3 & 20 \\
Redes sociales & 4 & 27 \\
Total & 15 & $100 \%$ \\
\hline
\end{tabular}

Nota. Elaboración propia en base a cuestionario aplicado a estudiantes embarazadas de Unidad Educativa Picoaza, Ecuador.

En esta interrogante las estudiantes indican con el 33 \% ocupar el tiempo en quehacer doméstico, otro importante grupo con el 27 \% indica utilizarlo en redes sociales, mientras que las actividades académicas y el ocio, obtienen el $20 \%$.

\section{Tabla 4}

Cuándo presenta algún tipo de dificultad a quien acude, dirigido a estudiantes embarazadas de la Parroquia Picoaza, Ecuador 2021

\begin{tabular}{llc}
\hline Ítems & $\mathbf{F}$ & $\%$ \\
\hline Con un familiar & 1 & 7 \\
Amigo & 6 & 40 \\
Pareja & 5 & 33 \\
Prefiere no comentarlo con nadie & 3 & 20 \\
Total & 15 & 100 \\
\hline Nota. Elaboración propia en base a cuestionario aplicado a estudiantes embarazadas de Unidad Educativa Picoaza, Ecuador.
\end{tabular}


En esta interrogante, con el $40 \%$ de estudiantes encuestados, mencionan que acuden donde un amigo cuando tienen alguna dificultad, lo que se considera muy de acuerdo en este ítem, puesto que al tener problemas buscan la manera de expresarlo con alguien. Mientras que con el $33 \%$ indican que acuden con su pareja para establecer soluciones, sin embargo, con el $20 \%$ prefiere no comentar con nadie en dificultad, lo que puede interpretarse como una situación en desacuerdo.

\section{Tabla 5}

Ha vivido una o varias de las siguientes situaciones, dirigido a estudiantes embarazadas de la Parroquia Picoaza, Ecuador 2021

\begin{tabular}{lcc}
\hline Ítems & F & \% \\
\hline Enfermedad de un familiar & 3 & 20 \\
Divorcio o separación de tus padres & 1 & 7 \\
Serios problemas económicos & 6 & 40 \\
Embarazos no deseados & 5 & 33 \\
Total & 15 & 100 \\
\hline
\end{tabular}

Nota: Elaboración propia en base a cuestionario aplicado a estudiantes embarazadas de Unidad Educativa Picoaza, Ecuador

El $40 \%$ de estudiantes embarazadas encuestadas indican haber estado con serios problemas económicos, mientras que con el 33 \% indican estar en una situación de embarazo no deseado, otro $20 \%$ menciona haber estado durante la enfermedad de un familiar muy querido y con el $7 \%$ indican haber vivido la situación de la separación de sus padres.

\section{Tabla 6}

Posee usted las siguientes características, dirigido a estudiantes embarazadas de la Parroquia Picoaza, Ecuador 2021

\begin{tabular}{lcc}
\hline Ítems & $\mathbf{F}$ & $\%$ \\
\hline Buenos sentimientos & 7 & 47 \\
Leal & 0 & 0 \\
Divertida & 6 & 40 \\
Reservada /cuidadosa & 2 & 13 \\
Total & 15 & 100 \\
\hline Nota: Elaboración propia en base a cuestionario aplicado a estudiantes embarazadas de Unidad Educativa Picoaza, Ecuador
\end{tabular}

Entre las características anteriores el $47 \%$ de estudiantes embarazadas mencionan tener buenos sentimientos lo que se percibe como muy bueno considerando la condición, mientras que con el $40 \%$ se consideran divertidas, lo que se puede dar a entender que no se refugian en un solo estado de ánimo, mientras que con el $13 \%$ se consideran cuidadosa o reservada. 


\section{Discusión}

La adolescencia puede ser comprendida como el período de evolución que ocurre de la niñez a la adultez, lo que Incluye algunos cambios, físicos y en la forma en que un joven se relaciona o interactúa con el entorno, en este sentido, para Ortega $(2011$, p. 5) "se considera que comienza alrededor de los 12 o 13 años y termina hasta los 19 o 20, durando casi una década. Comienza con la pubertad que es el proceso que conduce a la madurez sexual". No obstante, todo ese proceso tiene incidencia, por tanto, debe ser necesario la atención que se realice a adolescentes con el fin de disponer de un adecuado contexto escolar, tal como señala de Posada y Broche (2012, p. 233):

El éxito en un determinado contexto, como el escolar, transmite a las personas sentimientos positivos de autoestima y eficacia, que ayudan a tener la suficiente confianza para enfrentarse y salir airosos de las pruebas que la vida les depare en otros contextos.

El contexto educativo como proceso de formación implica una seria de interacciones que pueden ser reflejados en el comportamiento de cada individuo, es aquí donde se deben visualizar los posibles riegos, que impliquen acciones inmediatas por los entes de control debido a que las circunstancias de adversidad no son estáticas, sino que pueden cambian y para ello requieren estructuras en las conductas resilientes. Es importante determinar la efectividad de aplicar una escala de carácter resiliente para asegurar un cambio positivo en las personas que son objeto de estudio, tanto así que permitan identificar las variaciones del comportamiento con base en la situación de cada individuo:

Los factores de resiliencia de la escala SV-RES vinculados al rendimiento académico interactúan con otros factores protectores. Dichos factores apoyan el proceso de reelaboración de la adversidad. Uno de estos factores refiere a la forma como los adolescentes interpretan y desarrollan estrategias de acción para abordar la adversidad (Villalta, 2010, p. 180).

Por otro lado, "la resiliencia ha introducido una perspectiva diferente tanto en el ámbito de la psicopatología como en el de la educación al confiar más en la fortaleza de las personas y en sus posibilidades de realizar cambios" (Uriarte, 2005, p 61). Es así que al otorgar confianza en el individuo de poder superar la adversidad como ser humano con características de adaptabilidad en distintas condiciones.

Para Aveiga y Cedeño (2020, p. 431) "la resiliencia juega un papel importante en el proceso, por ser la habilidad que posee el ser humano para adaptarse de manera positiva a situaciones adversas". Por ende, se considera el potencial de cada individuo para ajustar su comportamiento y no acelerar un proceso natural que involucre interpretaciones erróneas de la percepción de cada persona, en este orden de ideas, Meza y Huamán (2019, p. 40), manifiestan que: "la resiliencia puede ser entendida también teniendo como estrategias la significación del arte, la danza, los lugares de memorialización. Pero, estos hechos no deben ser procesos aislados a la memoria colectiva, es necesario que sean acompañadas por otras medidas". 


\section{Conclusión}

En relación con los factores de resiliencia SV-RES respecto a estudiantes en estado de embarazo se evidencia la relación entre resiliencia y su condición pues existen situaciones que es necesario considerar para constituir las condiciones de dicha relación. Estas están afines al modelo protector de la resiliencia señalados en los factores F3 satisfacción F6 redes F9 afectividad considerados en las encuestas aplicadas.

Es evidente que existen factores que pueden considerarse preocupantes, pues en estos casos las estudiantes en condiciones de baja autoestima, o de alguna situación emocional, pueden no solo afectar su estado de salud, sino que también le afecta al hijo quien percibe las emociones de la futura madre.

Es importante mencionar, que las acciones desarrolladas por la profesional en Trabajo Social de la unidad educativa, son coordinadas con el equipo técnico profesional del Departamento de Consejería Estudiantil, quienes en conjunto implementan estrategias, que fomentan la autoestima en este grupo de estudiantes lo que incide en incrementar factores resilientes en estudiantes en estado de gestación.

\section{Referencias}

Aveiga Macay, V. R. y Cedeño Aguayo, E. L. (2020). Resiliencia de los adultos mayores del Centro Diurno Gerontológico "Cristo Rey" frente al abandono de sus familiares. Socialium, 4(2), 429-444. https://doi.org/10.26490/uncp.sl.2020.4.2.579

Balestena Sánchez, J., Pereda Serrano, Y. y Milán Soler, J. (2015). La edad materna avanzada como elemento favorecedor de complicaciones obstétricas y del nacimiento. Revista de Ciencias Médicas de Pinar del Río, 19(5), 789-802.

Barón, J. V. (2013). Embarazo en adolescentes complicaciones. Revista medica de Costa Rica y Centroamerica, 70(605), 65-69.

Binstock , G., y Náslund-Hadley , E. (2013). Maternidad adolescente y su impacto sobre las trayectorias educativas y laborales de mujeres de sectores populares urbanos de Paraguay. Papeles de Publación CIEAP/UAEM, (78), 15-40. http://www.scielo.org.mx/pdf/pp/v19n78/v19n78a3.pdf

Caballero Batista, A. y Méndez Díaz, T. (2005). El sentido psicologico del embarazo en adolescentes gestantes. Gale(108), 62.

https://go.gale.com/ps/anonymous?id=GALE\%7CA170020799\&sid=googleScholar\&v=2.1\&it=r\&l inkaccess=abs\&issn=00489115\&p=IFME\&sw=w

De Posada Rodríguez, S. y Broche Ulloa, M. (2012). Intervención educativa para fortalecer la resiliencia de madres adolescentes del Policlínico Vertientes. Humanidades Médicas, 12(2), 217-240. http://scielo.sld.cu/scielo.php?script=sci_arttext\&pid=S1727-81202012000200006 
Fernández Fernández, D., Altelarrea Soria, A. y Bravo Arroyo, M. (2002). El trabajo social en un equipo de orientación educativa y psicopedagógica. Red de información educativa. https://redined.mecd.gob.es/xmlui/handle/11162/92661

Gómez , I., Molina, R, y Zamberlin, N. (2010). Factores relacionados con el embarazo y la maternidad en menores de 15 años en América Latina y El Caribe. (L. T. Orozco, Ed.) Federación Latinoamericana de Sociedades de Obstetricia y Ginecología. http://www.codajic.org/sites/www.codajic.org/files/Factores\%20relacionados\%20\%20con\%20el \%20embarazo\%20y\%20la\%20maternidad\%20en\%20menores\%20de\%2015\%20a\%C3\%B1os\%20 ReporteFinalOct2010.pdf

León, P., Minassian, M., Borgoño, R. y Bustamante, F. (2008). Embarazo en adolescente. Revista pediátrica, 5(1), 42. http://www.revistapediatria.cl/volumenes/2008/vol5num1/5.html Martínez Sánchez, L., Jaramillo Jaramillo, L., Villegas Álzate, J., Álvarez Hernández, L. y Ruiz Mejía , C. (2018). La anemia fisiológica frente a la patológica en el embarazo. Revista Cubana de Obstetricia y Ginecología, 44(2). http://revginecobstetricia.sld.cu/index.php/gin/article/view/356/287

Meza Salcedo, A., y Huamán Tovar, E. (octubre de 2019). Desarrollo y ciudadanía: la resiliencia de las comunidades rurales en Pasco y Huánuco post conflicto armado en el Perú. Socialium, 4(1), 3449. https://doi.org/10.26490/uncp.sl.2020.4.1.508

Organización Mundial de la Salud, O. (2006). Las prioridades de salud sexual y reproductiva reciben un espaldarazo al más alto nivel. Revista Médica Electrónica de Portales Médicos.

Ortega Embrion , M. (2011). Identificar factores de protección y/o resiliencia del embarazo adolescente en mujeres de un contexto marginal urbano del municipio de La Paz [Archivo PDF]. CEDOC. http://cedoc.inmujeres.gob.mx/ftpg/BCS/bcs_meta1_2011.pdf

Posada, C. (2004). Embarazo en la adolescencia: no una opción, sino una falta de opciones. Revista Sexología y Sociedad, 10(25), 4-10. http://revsexologiaysociedad.sld.cu/index.php/sexologiaysociedad/article/view/268

Urbina, C. y Pacheco, J. (2006). Embarazo en adolescentes. Revista Peruana de Ginecología y Obstetricia, 52(1), 118-123. https://doi.org/10.31403/rpgo.v52i341

Uriarte Arciniega, J. (2005). La resiliencia. Una nueva perspectiva en psicopatología del desarrollo. Revista de Psicodidáctica, 10(2), 61-80. https://www.redalyc.org/pdf/175/17510206.pdf 
Villalta Páucar, M. A. (2010). Factores de resiliencia asociados al rendimiento académico en estudiantes de contextos de alta vulnerabilidad social. Revista de Pedagogía, 31(88), 159-188. https://www.redalyc.org/articulo.oa?id=65916617007

Villalta Páucar, M. A. (2010). Factores de resiliencia asociados al rendimiento académico en estudiantes de contextos de alta vulnerabilidad social. Revista de Pedagogía, 31(88), 159-188. https://www.redalyc.org/articulo.oa?id=65916617007

\section{Contribución de los autores}

TLZZ: Conceptualización, análisis formal, investigación, redacción: borrador original. EMAM: Metodología, supervición, validación, redacción: revisión y edición.

Fuentes de financiamiento

Autofinanciada.

\section{Conflictos de interés}

No presenta conflicto de intereses.

\section{Correspondencia}

tzamora1052@utm.edu.ec 